\title{
Spin-orbit splitting in non-relativistic and relativistic self-consistent models
}

\author{
M. López-Quelle, ${ }^{1, *}$ N. Van Giai, ${ }^{2}$ S. Marcos ${ }^{3}$ and L.N. Savushkin ${ }^{4}$ \\ ${ }^{1}$ Universidad de Cantabria, Departamento de Física Aplicada, 39005 Santander, Spain \\ 2 Institut de Physique Nucléaire, F-91406 Orsay Cedex, France \\ ${ }^{3}$ Universidad de Cantabria, Departamento de Física Moderna, 39005 Santander, Spain \\ ${ }^{4}$ St.-Petersburg Institute for Telecommunications, Department of Physics, 191065 St. Petersburg, \\ Russia
}

\begin{abstract}
The splitting of single-particle energies between spin-orbit partners in nuclei is examined in the framework of different self-consistent approaches, nonrelativistic as well as relativistic. Analytical expressions of spin-orbit potentials are given for various cases. Proton spin-orbit splittings are calculated along some isotopic chains $(\mathrm{O}, \mathrm{Ca}, \mathrm{Sn})$ and they are compared with existing data. It is found that the isotopic dependence of the relativistic mean field predictions is similar to that of some Skyrme forces while the relativistic Hartree-Fock approach leads to a very different dependence due to the strong non-locality.
\end{abstract}

PACS numbers: 21.10.Pc, 21.60.Jz

*e-mail address: lopezm@besaya.unican.es 


\section{INTRODUCTION}

One often asked question about the relativistic approach to nuclear structure is: what are the main physical effects that a relativistic approach can handle better than a non-relativistic approach? Among various arguments one feature seems to emerge quite obviously, namely the fact that, in a description based on the Dirac equation the nucleon spin degree of freedom is incorporated very naturally. Therefore, nuclear properties related to the spin and particularly spin-orbit effects in nuclei should come out from a relativistic description without any special adjustments whereas a non-relativistic approach would need ad hoc parameters. This was indeed so in the Dirac phenomenology analysis of spin observables measured in medium energy nucleon-nucleus scattering [1]. The interplay of two deep phenomenological potentials, the attractive scalar field and the repulsive vector field gives the right magnitude for the central and spin-orbit optical potentials.

However, if one tries to calculate microscopically the mean field experienced by a nucleon bound to a nucleus and predict spin-orbit splittings, it is not so clear how successful will be the various relativistic models. In this work, we would like to analyze the spin-orbit splittings calculated in different self-consistent models: non-relativistic Hartree-Fock with Skyrme-type forces, relativistic mean field theory (RMFT) and relativistic Hartree-Fock (RHF). Of course, a mean field approach like Hartree-Fock is designed for reproducing global ground state properties like total binding energies or densities but it is not supposed to describe correctly single-particle spectra because core polarization effects are known to modify importantly single-particle energies [2,3]. However, the core polarization effects should affect essentially in the same way the two members of a spin-orbit doublet if they are both below, or both above the Fermi level [4. Therefore, it is legitimate to study the question of spin-orbit splittings in Hartree-Fock or mean field frameworks for nuclei where both spin-orbit partners are occupied.

We carry out this study by looking at the evolution of the spin-orbit splittings of a $n l j_{>}-n l j_{<}$proton pair of states along an isotopic chain. The predictions of different models are compared with experimental values when they are available. The difficulties of such comparisons lie in the fact that the experimental information is scarce and it suffers sometimes of large uncertainties. This points to the necessity of having more data on spinorbit splittings if one aims at more quantitative conclusions about the validity of different models concerning their spin-orbit properties. Within these limitations, our analysis will be done for the $\mathrm{Ca}, \mathrm{O}$ and $\mathrm{Sn}$ isotopic chains.

The outline of the paper is as follows. In Section II and in the Appendix we derive the spin-orbit potentials corresponding to the different models: Skyrme-Hartree-Fock (SHF), RMFT and RHF. In Section III we discuss the results for the ${ }^{40} \mathrm{Ca}-{ }^{48} \mathrm{Ca}$ case as well as those concerning the $\mathrm{O}$ and $\mathrm{Sn}$ isotopes. Concluding remarks are made in Section IV.

\section{FORMALISM}

Let us derive the main expressions for the spin-orbit potentials in non-relativistic and relativistic approaches. In either case the general method is to rewrite the Hartree or HartreeFock equations for the single-particle states in a Schrödinger-like form and to identify a 
central potential and a spin-orbit potential. These potentials are local in the cases considered here, but they are generally state-dependent.

\section{A. Non-relativistic approach}

The SHF model is very simple because the Hartree-Fock equations for the single-particle wave functions $\phi_{i}$ and energies $\varepsilon_{i}$ take the form of a Schrödinger equation with an effective mass $m^{*}(\mathbf{r})$ and a local, state-independent potential. All non-locality effects are described by this effective mass and consequently, the SHF equations contain also first derivatives of the wave function. It is natural to introduce an asymptotically equivalent wave function [5]:

$$
\tilde{\phi}_{i}(\mathbf{r})=\left(\frac{m^{*}(\mathbf{r})}{m}\right)^{-\frac{1}{2}} \phi_{i}(\mathbf{r})
$$

which now satisfies a Schrödinger equation with a constant mass $m$ and a state-dependent local potential:

$$
V\left(\varepsilon_{i}, \mathbf{r}\right)=V_{0}\left(\varepsilon_{i}, \mathbf{r}\right)+V_{L S}(\mathbf{r}) \mathbf{l} . \mathbf{s} .
$$

The spin-orbit potential is (for spherical symmetry):

$$
V_{L S}(q, r)=\frac{m_{q}^{*}(r)}{m}\left[\frac{w}{r}\left[\rho^{\prime}(r)+\rho_{q}^{\prime}(r)\right]-\frac{1}{4 r}\left[\left(t_{1} x_{1}+t_{2} x_{2}\right) J(r)+\left(t_{2}-t_{1}\right) J_{q}(r)\right]\right],
$$

where $w, t_{i}, x_{i}$ are Skyrme force parameters, $\rho_{q}$ and $J_{q}$ are nucleon densities and spin densities $(q=n$ or $p)$ with $\rho=\rho_{n}+\rho_{p}$ and $J=J_{n}+J_{p}$ [5]. The spin densities are practically zero in spin-saturated nuclei but they can give some contributions in spin-unsaturated ones. The above expression shows that $V_{L S}$ is surface peaked, and that proton spin-orbit splittings $\Delta_{L S}$ should have little isotopic dependence in the SHF model as we shall see in the next sections. One should, however, keep in mind that another contribution to $\Delta_{L S}$ comes from the energy-dependent central potential. One can easily check that the energy dependence of $V_{0}\left(\varepsilon_{i}, \mathbf{r}\right)$ is entirely contained in a term $\left(1-m^{*}(\mathbf{r}) / m\right) \varepsilon_{i}$ [5].

Recently, some attempts have been made in order to introduce more freedom in the spinorbit term of the Skyrme parametrization with the aim of improving isotope shift predictions in the $\mathrm{Pb}$ region [6]. The authors of Ref. [6] modify directly the Skyrme energy functional so that the first term of Eq.(3) becomes $w \rho^{\prime}(r) / r+w^{\prime} \rho_{q}{ }^{\prime}(r) / r$ and the spin density terms are dropped. It is found that a better description of data is reached when $w^{\prime}$ is nearly $-w$ (parameter set SkI4), i.e., when the proton spin-orbit potential depends almost entirely on the derivative of the neutron density.

\section{B. Relativistic approach}

Let us now calculate the spin-orbit potential in the general RHF case. The corresponding expressions for the RMFT case will be easily obtained by dropping all contributions corresponding to exchange (Fock) terms. Starting from the RHF equation for the 4-component spinors we shall obtain a Schrödinger-like equation for the upper component which allows 
us to identify the central and spin-orbit potentials. We use the notations introduced in Ref. |7].

We start from Eq.(C3) of Ref. [7] for the spinor $\left(G_{i}, F_{i}\right)$ :

$$
\begin{aligned}
G_{i}^{\prime} & =\left(-\frac{\kappa_{i}}{r}-\Sigma_{T, i}-P_{i}\right) G_{i}+\left(m+E_{i}+\Sigma_{S, i}-\Sigma_{0, i}-Q_{i}\right) F_{i} \\
F^{\prime} & =\left(m-E_{i}+\Sigma_{S, i}+\Sigma_{0, i}+R_{i}\right) G_{i}+\left(\frac{\kappa_{i}}{r}+\Sigma_{T, i}+S_{i}\right) F_{i},
\end{aligned}
$$

where the $\Sigma$ 's are the direct (Hartree) self-energies whereas $P, Q, R, S$ are related to the exchange (Fock) contributions. We shall drop the state indices $i$ from now on. The first step is to eliminate the lower component $F$ and obtain a second order differential equation for the upper component $G$ :

$$
\Lambda G^{\prime \prime}+\alpha_{1} G^{\prime}+\alpha_{0} G=0
$$

where

$$
\begin{aligned}
\Lambda= & \left(m+E+\Sigma_{S}-\Sigma_{0}-Q\right)^{-1} \\
\alpha_{1}= & \Lambda^{\prime}+\Lambda(P-S) \\
\alpha_{0}= & \Lambda^{\prime}\left(\frac{\kappa}{r}+\Sigma_{T}+P\right)+\Lambda\left(-\frac{\kappa}{r^{2}}+\Sigma_{T}^{\prime}+P^{\prime}\right) \\
& -\left(M-E+\Sigma_{S}+\Sigma_{0}+R\right)-\Lambda\left(\frac{\kappa}{r}+\Sigma_{T}+P\right)\left(\frac{\kappa}{r}+\Sigma_{T}+S\right) .
\end{aligned}
$$

Next, we look for a modified function $\tilde{G}$ :

$$
G=\lambda \tilde{G},
$$

such that $G$ and $\tilde{G}$ are identical asymptotically while the differential equation satisfied by $\tilde{G}$ contains no first derivative. This condition determines $\lambda$ to be:

$$
\lambda=C \Lambda^{-\frac{1}{2}} e^{-\frac{1}{2} \int(P-S) d r} .
$$

where the constant $\mathrm{C}$ is determined from the condition $\int \tilde{G}^{2} d r=1$. In the limit of RMFT the exchange quantities $P$ and $S$ are zero and one recovers the familiar result $\lambda=\Lambda^{-\frac{1}{2}}$.

We can now write the differential equation satisfied by $\tilde{G}$ in the form:

$$
-\frac{\hbar^{2}}{2 m}\left(\tilde{G}^{\prime \prime}+\left[\frac{\lambda^{\prime \prime}}{\lambda}-\frac{1}{2}\left(\frac{\alpha_{1}}{\Lambda}\right)^{2}+\frac{\alpha_{0}}{\Lambda}\right] \tilde{G}\right)=0 .
$$

The potential in state $i$ can be identified from the coefficient of $\tilde{G}$. It is possible to separate out the spin-orbit part $V_{L S}$ of this potential by observing that the quantities $\Lambda, P, Q, R, S$ depend on $\kappa \equiv(2 j+1)(l-j)$ (see, e.g., Ref. [0]) which in turn can be expressed in terms of $\langle\mathbf{l} . \mathbf{s}\rangle$. The complete expressions for $V_{L S}$ are given in the Appendix.

\section{EVOLUTION OF SPLITTINGS ALONG ISOTOPIC CHAINS}

We now turn to an analysis of spin-orbit splittings in some finite nuclei. We concentrate on the quantities:

$$
\Delta\left(n l j_{<}-n l j_{>}\right) \equiv \varepsilon_{n l j_{<}}-\varepsilon_{n l j_{>}}
$$




\section{A. Ca isotopes}

In $\mathrm{Ca}$ isotopes, the experimental situation concerning the $1 d_{5 / 2}$ and $1 d_{3 / 2}$ proton states is not very accurately established. In ${ }^{40} \mathrm{Ca}$, the generally accepted value of $1 d_{3 / 2}$ singleparticle energy is $\varepsilon_{1 d 3 / 2}=-8.3 \mathrm{MeV}$ [8], [9], [10] However, the values of $\varepsilon_{1 d 5 / 2}$ are much more dispersed: $-15.1 \mathrm{MeV}$ [11], $-15.5 \mathrm{MeV}$ [12], $-16 \mathrm{MeV}$ [9], $-14.3 \mathrm{MeV}$ [10]. Note that the most recent values of Ref. [10] have been obtained as the centroid energies of the singleparticle spectroscopic strength distributions:

$$
\varepsilon_{n l j} \equiv \frac{\sum_{i} E_{i} S_{i}}{\sum_{i} S_{i}}
$$

and therefore, they take into account the strong fragmentation of the single-particle states. The fragment energies $E_{i}$ and spectroscopic factors $S_{i}$ were taken from Refs. [13], [14], [15], [16], [17] and [18]. The corresponding experimental spin-orbit splittings of ${ }^{40} \mathrm{Ca}$ are plotted on Fig. 1. In ${ }^{48} \mathrm{Ca}$, the experimental proton energies $\varepsilon_{1 d 3 / 2}$ are respectively $-16.2 \mathrm{MeV}$ 9] and $-15.5 \mathrm{MeV}$ [10] whereas the $\varepsilon_{1 d 5 / 2}$ energies are $-21.5 \mathrm{MeV}$ [9] and $-20.5 \mathrm{MeV}$ [10]. This gives the spin-orbit energies in ${ }^{48} \mathrm{Ca}$ shown in Fig. 1.

At this point we must mention that some theoretical papers, e.g., Ref. [7] quote a somewhat smaller value for the experimental $\Delta_{S . O .}(1 d 3 / 2-1 d 5 / 2)$ in ${ }^{48} \mathrm{Ca}$ based on an earlier paper [19] but the experimental source remains uncertain.

In Fig. 11 we display the results calculated with the following models: SHF with a standard SIII force and with the SkI4 parametrization of Ref. [6], RMFT with the commonly used NL-SH 20] and NL3 21] parametrizations, RHF with $(\sigma, \omega, \rho, \pi)$ mesons (model e) of Ref. [7]). The two parametrizations NL-SH and NL3 have been chosen as the most representative and successful in describing nuclear ground states in a wide range of nuclei.

All calculated results for $\Delta\left(1 d_{3 / 2}-1 d_{5 / 2}\right)$ show a linear A-dependence between $A=40$ to $A=48$. However, whereas the values of SHF and RMFT models decrease only slightly those of the RHF model exhibit a large decrease.

The relatively small variations of the SIII results can be understood by examining the $V_{L S}$ potential of Eq.(3). When going from ${ }^{40} \mathrm{Ca}$ to ${ }^{48} \mathrm{Ca}$ one acquires extra neutron densities $\rho_{7 / 2}(r)$ and $J_{7 / 2}(r)$ due to the filling of the $1 f_{7 / 2}$ orbital while the core neutron and proton densities do not change much. For the SIII parametrization, $x_{1}=x_{2}=0$ so that $J_{7 / 2}(r)$ gives no contribution to $V_{L S}(p, r)$ whereas $\rho_{7 / 2}^{\prime}(r)$ yields a contribution with a node at the surface, i.e., a small effect on $\Delta\left(1 d_{3 / 2}-1 d_{5 / 2}\right)$. The same analysis holds for the SkI4 results. For the RMFT case the interpretation of results is also simple. The spin-orbit potential reduces to the first term of Eq.(A4), i.e., $-2 \Lambda^{\prime} / \Lambda r$. With the definition of $\Lambda$ given by Eq.(6) it is seen that the variation of spin-orbit potential is obtained by folding the derivative of the scalar and vector $1 f_{7 / 2}$ densities with the (short-ranged) $\sigma$ and $\omega$ form factors and therefore, it has a node near the surface and the variation of spin-orbit splitting must be small. We should note, however, that the central potential that one can identify from Eq.(9) has some state dependence due to the effective mass $m^{*}=m+\Sigma_{S}$ and this effect can also contribute to the energy splitting. Furthermore, the kinetic energy and the central potential contribute also to this splitting through the j-dependence of the radial part in the wave function.

The RHF results are more difficult to interpret. From Eq.(9) we identify the kinetic energy $T$, central potential $V_{0}$ and spin-orbit potential $V_{L S}$ such that: 


$$
\langle T\rangle_{i}+\left\langle V_{0}\right\rangle_{i}+\left\langle V_{L S}\right\rangle_{i}=\varepsilon_{i}\left(1+\frac{\varepsilon_{i}}{2 m}\right)
$$

The expressions for $V_{L S}$ given in the Appendix are complicated. The non-locality of the original RHF mean field induces a strong state dependence in the local equivalent potentials and therefore, variations with $j_{>}$and $j_{<}$will be found in all three terms of Eq.(12). This is

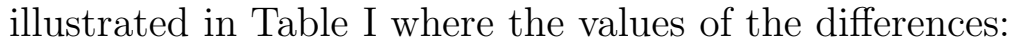

$$
\begin{aligned}
\Delta T & \equiv\langle T\rangle_{1 d 3 / 2}-\langle T\rangle_{1 d 5 / 2}, \\
\Delta V_{0} & \equiv\left\langle V_{0}\right\rangle_{1 d 3 / 2}-\left\langle V_{0}\right\rangle_{1 d 5 / 2}, \\
\Delta V_{L S} & \equiv\left\langle V_{L S}\right\rangle_{1 d 3 / 2}-\left\langle V_{L S}\right\rangle_{1 d 5 / 2}
\end{aligned}
$$

are shown. In ${ }^{40} \mathrm{Ca}$ the calculated spin-orbit splitting is practically equal to $\Delta V_{L S}$, with a strong cancellation of the two other terms. In ${ }^{48} \mathrm{Ca} \Delta V_{L S}$ is only one third of $\varepsilon_{1 d 3 / 2}-\varepsilon_{1 d 5 / 2}$, the rest coming from an increase of $\Delta V_{0}$ and $\Delta T$.

It is possible to analyze further the respective role of the isovector $\pi$ and $\rho$ mesons in the decrease of $\Delta\left(1 d_{3 / 2}-1 d_{5 / 2}\right)$ from ${ }^{40} \mathrm{Ca}$ to ${ }^{48} \mathrm{Ca}$. The key effect comes from the $\pi$ meson and the strong non-locality related to its light mass. If one artificially increases $m_{\pi}$ up to $600 \mathrm{MeV}$ (and renormalize accordingly the $f_{\pi}$ coupling constant to keep reasonable singleparticle levels) one tends to a more local situation and the strong decrease of $\Delta\left(1 d_{3 / 2}-1 d_{5 / 2}\right)$ vanishes. The role of the $\rho$ meson can be studied by repeating the calculations without its contributions to the Fock terms. It is found that the decrease of $\Delta\left(1 d_{3 / 2}-1 d_{5 / 2}\right)$ still remains. 


\section{B. Oxygen isotopes}

The $\mathrm{O}$ chain is particularly interesting because of the prospects of reaching experimentally new, unstable isotopes with a large neutron excess. We examine here the evolution of the $1 p_{1 / 2}-1 p_{3 / 2}$ splitting of proton levels. In Fig. 2 we summarize the results obtained with SHF (SIII and SkI4 parametrizations), RMFT (NL-SH and NL3 parametrizations) and RHF (model $e$ ) of Ref. [7]). We note that the results of NL-SH and NL3 are rather similar for spin-orbit splittings in $\mathrm{O}$ isotopes. This behaviour remains also in the Sn isotopes (see next subsection). These two parametrizations differ mostly by their predicted compression modulus $\mathrm{K}$ and therefore, this indicates that $\mathrm{K}$ has little effect on spin-orbit properties in the RMFT. One can distinguish three intervals ending at $A=22,24$ and 28 which correspond to the filling of $1 d_{5 / 2}, 2 s_{1 / 2}$ and $1 d_{3 / 2}$ subshells, respectively. The trend in the $1 d_{5 / 2}$ subshell resembles that of the $\mathrm{Ca}$ isotopes, namely a relatively small variation of $\Delta\left(1 p_{1 / 2}-1 p_{3 / 2}\right)$ for the SHF and RMFT models and a large decrease for the RHF model. A similar discussion as for the $\mathrm{Ca}$ isotopes can be done here. It would be interesting to measure experimentally the variations of spin-orbit splittings in the chain of Oxygen isotopes. For the isotopes heavier than $A=22$ RMFT shows a maximum at $A=24$ followed by a decrease whereas for RHF the behaviour is opposite, with an increase of the spin-orbit splitting after $A=22$.

\section{Sn isotopes}

In the chain of $\mathrm{Sn}$ isotopes proton spin-orbit splittings are experimentally known in several nuclei [22]. In Fig. 3 are shown the calculated and measured values of $\Delta\left(2 p_{1 / 2}-2 p_{3 / 2}\right)$ for protons, and in Fig. 4 are displayed the calculated results for $\Delta\left(1 f_{5 / 2}-1 f_{7 / 2}\right)$. From Fig. 3 it can be seen that none of the models is able to reproduce the very small empirical values of $\Delta\left(2 p_{1 / 2}-2 p_{3 / 2}\right)$. In the range $\mathrm{A}=112-124$ the SIII results show relatively small variations whereas the 3 other models give large fluctuations. Of course, it would be more satisfactory to calculate these nuclei using a Hartree-Fock-BCS description. It is not clear, however, that the proton spin-orbit splittings would be significantly affected so as to bring them into agreement with the data. Indeed, in Ref. [23] a HF-BCS was performed for Sn isotopes using the effective interaction SkI4 and a density-dependent pairing force. The values of $\Delta\left(2 p_{1 / 2}-2 p_{3 / 2}\right)$ thus obtained vary from $1.6 \mathrm{MeV}$ in ${ }^{112} \mathrm{Sn}$ to $2.0 \mathrm{MeV}$ in ${ }^{124} \mathrm{Sn}$. As for the $1 f_{5 / 2}-1 f_{7 / 2}$ splitting, Fig. 1 shows a wide range of predictions. For instance, in $\mathrm{A}=100 \mathrm{RHF}$ is as low as $2.2 \mathrm{MeV}$ while the other models are in the $5-6 \mathrm{MeV}$ range . It would be very interesting to have data on this $1 f_{5 / 2}-1 f_{7 / 2}$ case in order to shed light on this issue.

\section{CONCLUSION}

We have examined in this work some predictions of spin-orbit splittings in the framework of non-relativistic and relativistic mean field approaches. We selected, as a representative case of the non-relativistic self-consistent approach, the Skyrme-Hartree-Fock model because it is widely used and its analytic simplicity lends itself to an easy interpretation of the calculated splittings. Numerical results were obtained with two versions of the effective 
Skyrme interaction, the standard parametrization SIII and the more recently proposed SkI4 which contains more degrees of freedom in its spin-orbit part. On the relativistic side, the RMFT is also a successful model for describing nuclear ground states and we have examined its spin-orbit predictions with the often used non-linear versions NL-SH and NL3. Here also the analytic form of the one-body spin-orbit potential is simple enough to allow some insight into the numerical results. In addition, we have included in our study the RHF model of Ref. [7] in order to examine the role of the pion in the spin-orbit splittings.

One conclusion which can be drawn is that there is no clear advantage of the RMFT over the non-relativistic Skyrme-Hartree-Fock approach. It is true that in RMFT, the spin-orbit properties come out together with the central part of the mean field whereas in the nonrelativistic approach one has the freedom of one or more additional spin-orbit parameters. Nevertheless, it cannot be concluded that the RMFT spin-orbit splittings describe the data particularly well. In Refs. [24, [25] an interesting formal connection is made between RMFT and Skyrme-HF, but we can see here that, at a quantitative level, not only their spin-orbit predictions do differ, but even between SIII and SkI4 there are sizable differences in $\Delta_{\text {S.O. }}$.

All models fail to reproduce the very small $2 p_{1 / 2}-2 p_{3 / 2}$ proton splittings in Sn isotopes and it does not seem that pairing correlations can improve this prediction. As for the RHF approach, the light pion mass produces strongly non-local Fock fields, i.e., a strong state dependence of the mean fields. Consequently, the splitting between spin-orbit partners is due not only to $\left\langle V_{L S} \mathbf{l} . \mathbf{s}\right\rangle_{l j}$ but also to a large extent to the state dependence of the central potential. Thus, the evolution of the predicted splittings along an isotopic chain does not resemble that of less non-local models like RMFT or Skyrme. It appears that the isotopic dependence of $\Delta$ disagrees with the data in the case of RHF.

The conclusion made earlier [7] that RHF describes well the experimental $\Delta_{S . O}$. both in ${ }^{40} \mathrm{Ca}$ and ${ }^{48} \mathrm{Ca}$ was too premature in view of the uncertainties in the experimental values.

Finally, the question of spin-orbit predictions in the framework of self-consistent theories is still open. Existing parametrizations of effective interactions, relativistic as well as nonrelativistic, need further improvements. The RMF predictions seem to follow a common trend whereas the Skyrme-HF results may differ somewhat depending on the parametrizations, as it can be seen with SIII and SkI4 in Sn isotopes. Improving the parametrizations necessitates better comparisons with experiment. In this respect, it would be very helpful to have more data on the evolution of spin-orbit splittings along isotopic chains. Without better data, it is not possible at the moment to state that the spin-orbit problem is understood either by RMFT or by the non-relastivistic approach.

\section{ACKNOWLEDGMENTS}

We would like to thank M. Bender and R. Wyss for their comments and A.M. Oros for the data on $\mathrm{Ca}$ isotopes.

This work was supported in part by contract DGICYT PB97-0360 (Spain). 


\section{APPENDIX A:}

In this Appendix we give the expressions for $V_{L S}$ in the general case of RHF. The special case of RMFT is easily obtained by setting all functions $P, Q, R, S$ to zero. The notations are those of Ref. [7].

Eq.(9) is a Schrödinger-type equation for a state of angular momentum $(l, j)$. We split the coefficient of $\tilde{G}$ into a centrifugal term, a central potential and a spin-orbit potential:

$$
\frac{\lambda^{\prime \prime}}{\lambda}-\frac{1}{2}\left(\frac{\alpha_{1}}{\Lambda}\right)^{2}+\frac{\alpha_{0}}{\Lambda} \equiv \frac{-l(l+1)}{r^{2}}-\frac{2 m}{\hbar^{2}}\left[V_{0}(r)+V_{L S}(r)\langle\mathbf{l} . \mathbf{s}\rangle_{l j}-\varepsilon(1+\varepsilon / 2 m)\right] .
$$

The functions $P, Q, R, S$ can be expressed as second order polynomials in $\kappa$, namely, $P=$ $P_{0}+P_{1} k+P_{2} k^{2}$ and similarly for $Q, R, S$. Here $\kappa=(2 j+1)(l-j)=\frac{1}{2} \Omega(2 j+1)$ where $\Omega=+1$ if $j=l-\frac{1}{2}$ and $\Omega=-1$ if $j=l+\frac{1}{2}$. It is easy to relate powers of $\kappa$ to $\langle\mathbf{1 . s}\rangle_{l j}$. For instance, we have:

$$
2\langle\mathbf{l} . \mathbf{s}\rangle_{l j}=-\hbar^{2}(1+\kappa) .
$$

Thus, all contributions to $V_{L S}(r)$ can be identified by expressing the $\kappa$-dependence into a $\langle\mathbf{l} . \mathbf{s}\rangle_{l j}$-dependence. One then obtains:

$$
V_{L S}(r)=V_{L S}^{(I)}+V_{L S}^{(I I)}+V_{L S}^{(I I I)}
$$

where

$$
\begin{aligned}
V_{L S}^{(I)}= & \frac{1}{m}\left\{-\frac{\Lambda^{\prime}}{\Lambda}\left(\frac{1}{r}+P_{1}\right)+\frac{1}{r^{2}}-P^{\prime}+\frac{R_{1}}{\Lambda}+\left(\frac{1}{r}+P_{1}\right)\left(\Sigma_{T}^{D}+S_{0}\right)+\left(\frac{1}{r}+S_{1}\right)\left(\Sigma_{T}^{D}+P_{0}\right)\right. \\
+ & \frac{\Lambda^{\prime}}{\Lambda} P_{2}+P^{\prime}{ }_{2}-\frac{R_{2}}{\Lambda}-\left(\Sigma_{T}^{D}+P_{0}\right) S_{2}-\left(\frac{1}{r}+P_{1}\right)\left(\frac{1}{r}+S_{1}\right)-P_{2}\left(\Sigma_{T}^{D}+S_{0}\right) \\
+ & {\left.[l(l+1)+1]\left[\left(\frac{1}{r}+P_{1}\right) S_{2}+\left(\frac{1}{r}+S_{1}\right) P_{2}\right]-[2 l(l+1)+1] P_{2} S_{2}\right\} } \\
V_{L S}^{(I I)}= & \frac{1}{m}\left\{-\frac{\Lambda^{\prime}}{\Lambda}\left(P_{1}-S_{1}\right)-\left(P_{0}-S_{0}\right)\left(P_{1}-S_{1}\right)\right. \\
& +\frac{\Lambda^{\prime}}{\Lambda}\left(P_{2}-S_{2}\right)+\frac{1}{2}\left(P_{1}-S_{1}\right)^{2}+\left(P_{0}-S_{0}\right)\left(P_{2}-S_{2}\right) \\
& \left.-[l(l+1)+1]\left(P_{1}-S_{1}\right)\left(P_{2}-S_{2}\right)+[2 l(l+1)+1] \frac{1}{2}\left(P_{2}-S_{2}\right)^{2}\right\} \\
& \left.-[l(l+1)+1] \frac{1}{2}\left(P_{1}-S_{1}\right)\left(P_{2}-S_{2}\right)+[2 l(l+1)+1] \frac{1}{4}\left(P_{2}-S_{2}\right)^{2}\right\} \\
& +\frac{1}{2 \Lambda}\left(P_{2}-S_{2}\right)-\frac{1}{2}\left(P^{\prime}{ }_{2}-S_{2}^{\prime}\right)+\frac{1}{4}\left(P_{1}-S_{1}\right)^{2}+\frac{1}{2}\left(P_{0}-S_{0}\right)\left(P_{2}-S_{2}\right) \\
V_{L S}^{(I I I)}= & \frac{\Lambda^{\prime}}{2 \Lambda}\left(P_{1}-S_{1}\right)+\frac{1}{2}\left(P^{\prime}{ }_{1}-S^{\prime}{ }_{1}\right)-\frac{1}{2}\left(P_{0}-S_{0}\right)\left(P_{1}-S_{1}\right)
\end{aligned}
$$




\section{REFERENCES}

[1] B.C. Clark, R.L. Mercer and P. Schwandt, Phys. Lett. 122B, 211 (1983).

[2] I. Hamamoto and P. Siemens, Nucl. Phys. A269, 199 (1976).

[3] V. Bernard and N. Van Giai, Nucl. Phys. A348, 75 (1980).

[4] K. Rutz, M. Bender, P. -G. Reinhard, J. A. Marhun and W. Greiner, Nucl. Phys. A634, 67 (1998).

[5] M. Beiner, H. Flocard, N. Van Giai and Ph. Quentin, Nucl. Phys. A238, 29 (1975).

[6] P. -G. Reinhard and H. Flocard, Nucl. Phys. A584, 467 (1995).

[7] A. Bouyssy, J.F. Mathiot, N. Van Giai and S. Marcos, Phys. Rev. C 36, 380 (1987).

[8] J.H.E. Mattauch, W. Thiele and A.H. Wapstra, Nucl. Phys. 67, 32 (1965).

[9] L. Ray and P.E. Hodgson, Phys. Rev. C 20, 2403 (1979).

[10] A.M. Oros, Ph.D. thesis, University of Köln, unpublished (1996).

[11] A. Swift and L.R.B. Elton, Phys. Rev. Lett. 17, 484 (1966).

[12] H.Tyren, S. Kullander, O. Sundberg, R. Ramachandran, P. Isacsson and T. Berggren, Nucl. Phys. A79, 321 (1966).

[13] Database of the National Nuclear Data center, Brookhaven.

[14] P.M. Endt, Nucl. Phys. A521, 1 (1990).

[15] D.H. Youngblood, R.L. Kozub, R.A. Kenefick, J.C. Hiebert, Phys. Rev. C 2, 477 (1970).

[16] B.W. Devins. D.L. Friesel, W.P. Jones, A.C. Attard, S.F. Collins, G.G. Shute, B.M. Spicer, V.C. Officer, I.D. Svalbe, R.S. Henderson, W.E. Dellhopf, Phys. Rev. C 24, 59 (1981).

[17] F.J. Eckle, H. Lenske, G. Eckle, G. Graw, R. Hertenberger, H. Kader, H.J. Maier, F. Merz, H. Nann, P. Schiemenz and H.H. Wolter, Nucl. Phys. A506, 159 (1990).

[18] P. Doll, G.J. Wagner, K.T. Knöpfle and G. Mairle, Nucl. Phys. A263, 210 (1976).

[19] X. Campi and D.W.L. Sprung, Nucl. Phys. A194, 401 (1972).

[20] M.M. Sharma, M.A. Nagarajan and P. Ring, Phys. Lett. B 312, 377 (1993).

[21] G.A. Lalazissis, J. König and P. Ring, Phys. Rev. C 55, 540 (1997).

[22] N.B. Gove and A.H. Wapstra, Nucl. Data Tables 11, 127 (1972);

A. Warwick, R. Chapman, J.L. Durell and J.N. Mo, Nucl. Phys. A391, 9 (1982).

[23] Y. Shen and Z. Ren, Z. Phys. A 356, 133 (1996).

[24] A. Bouyssy and S. Marcos, Phys. Lett. 127B, 157 (1983).

[25] P.-G. Reinhard Rep. Prog. Phys. 52, 515 (1989). 


\section{TABLES}

TABLE I. The quantities $\Delta T, \Delta V_{0}, \Delta V_{L S}$ and $\varepsilon_{1 d 3 / 2}-\varepsilon_{1 d 5 / 2}$ calculated with RHF for protons in ${ }^{40} \mathrm{Ca}$ and ${ }^{48} \mathrm{Ca}$ (in $\mathrm{MeV}$ ).

\begin{tabular}{ccccc}
\hline \hline Nucleus & $\Delta T$ & $\Delta V_{0}$ & $\Delta V_{L S}$ & $\varepsilon_{1 d 3 / 2}-\varepsilon_{1 d 5 / 2}$ \\
\hline${ }^{40} \mathrm{Ca}$ & -2.94 & 3.31 & 7.54 & 8.01 \\
${ }^{48} \mathrm{Ca}$ & -1.73 & 4.25 & 1.38 & 4.06 \\
\hline \hline
\end{tabular}




\section{FIGURES}

FIG. 1. The $\Delta\left(1 d_{3 / 2}-1 d_{5 / 2}\right)$ proton splittings in Ca isotopes calculated with SIII (dot-dashed line), SkI4 (long-dashed line), RMFT (dotted line) and RHF (full line) models. Experimental values in $\mathrm{A}=40$ and $\mathrm{A}=48$ are shown: $\mathrm{A}$ [10], $\mathrm{B}$ [11], $\mathrm{C}$ [8] and $\mathrm{D}$ [9].

FIG. 2. The $\Delta\left(1 p_{1 / 2}-1 p_{3 / 2}\right)$ proton splittings in O isotopes calculated with SIII (dot-dashed line), SkI4 (long-dashed line), RMFT (dotted line) and RHF (full line) models. The experimental value for $\mathrm{A}=16$ is indicated.

FIG. 3. The $\Delta\left(2 p_{1 / 2}-2 p_{3 / 2}\right)$ proton splittings in Sn isotopes calculated with SIII (dot-dashed line), SkI4 (long-dashed line), RMFT (dotted line) and RHF (full line) models. Experimental values for $\mathrm{A} \geq 112$ are shown.

FIG. 4. The $\Delta\left(1 f_{5 / 2}-1 f_{7 / 2}\right)$ proton splittings in Sn isotopes calculated with SIII (dot-dashed line), SkI4 (long-dashed line), RMFT (dotted line) and RHF (full line) models. 


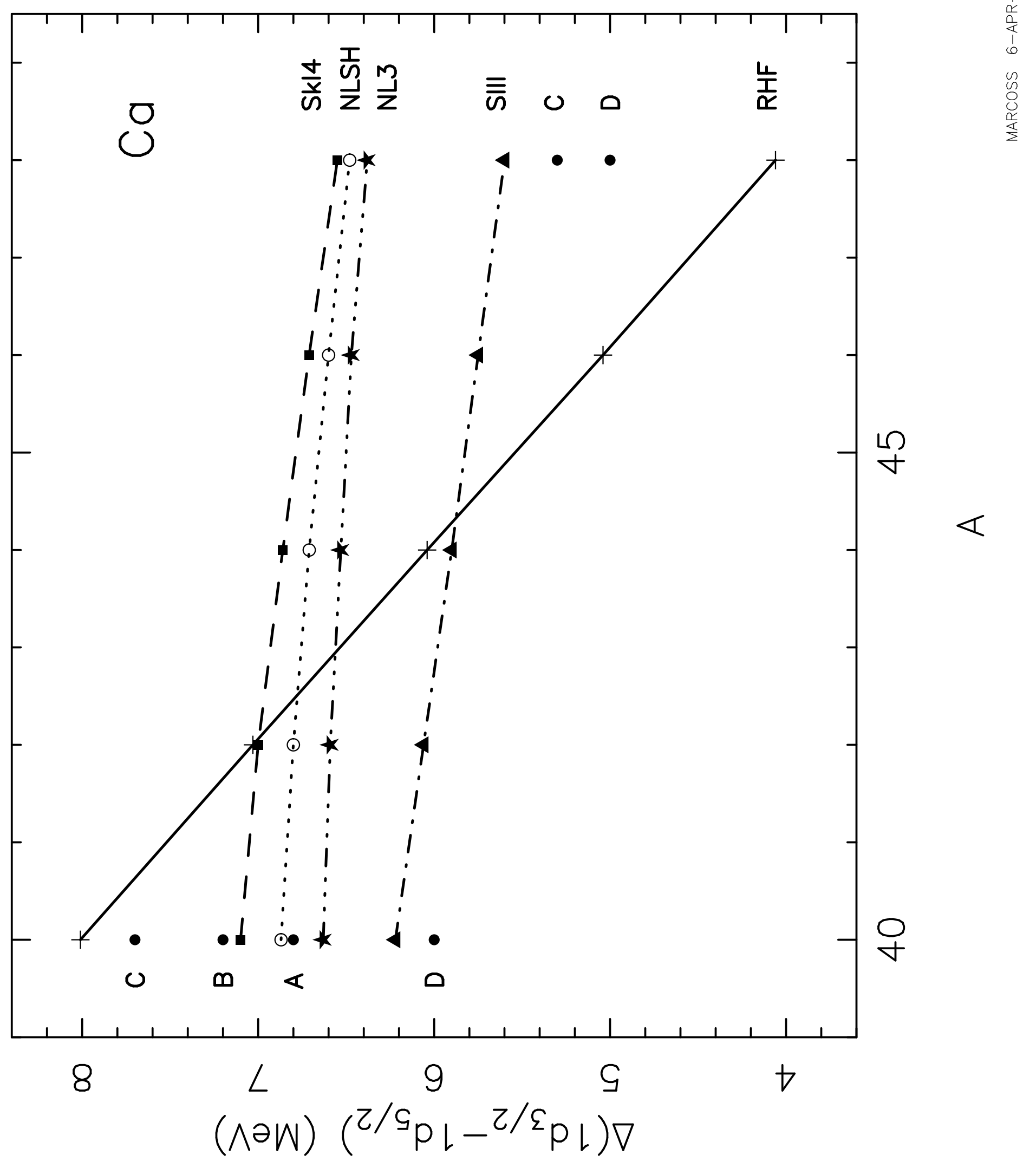




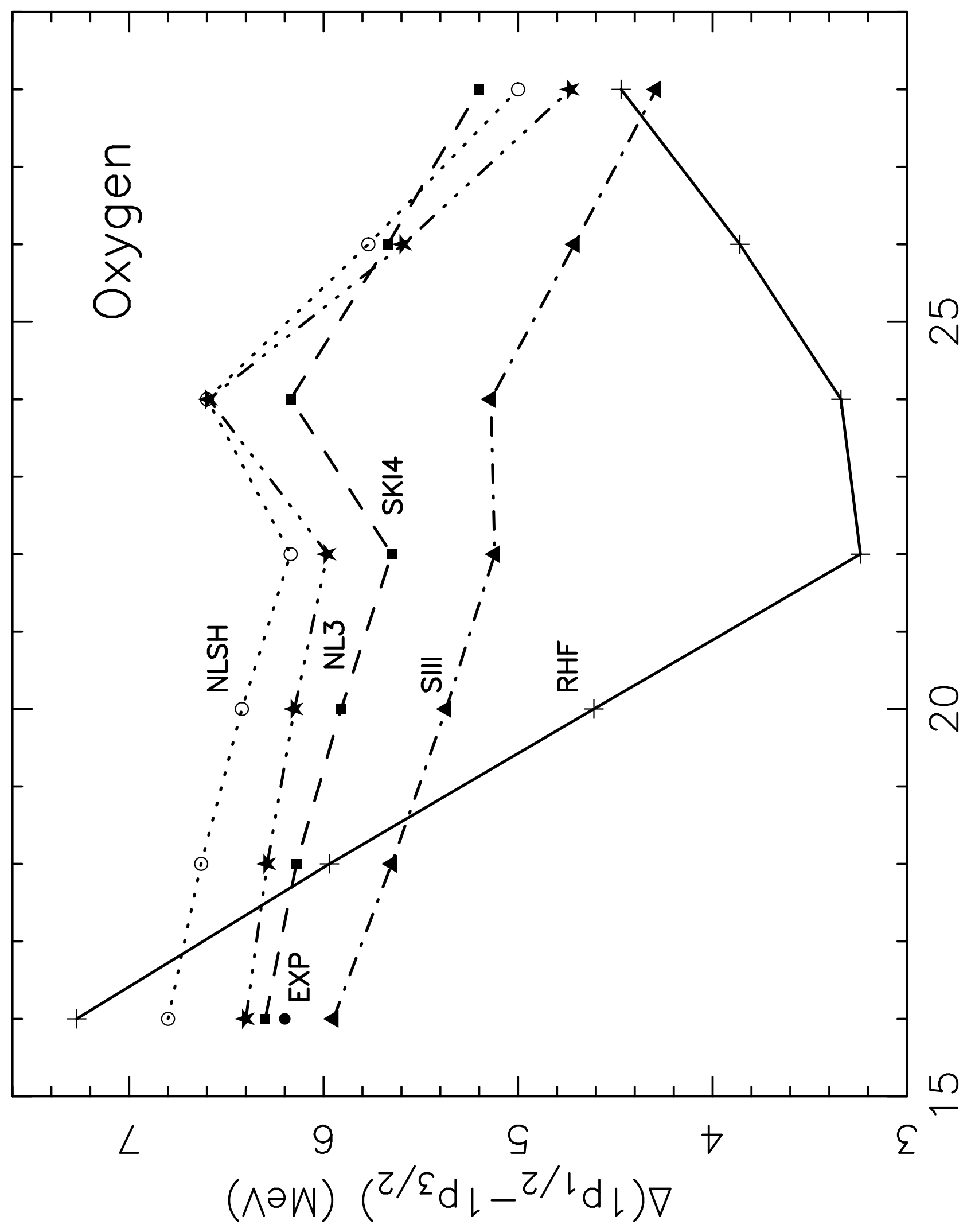




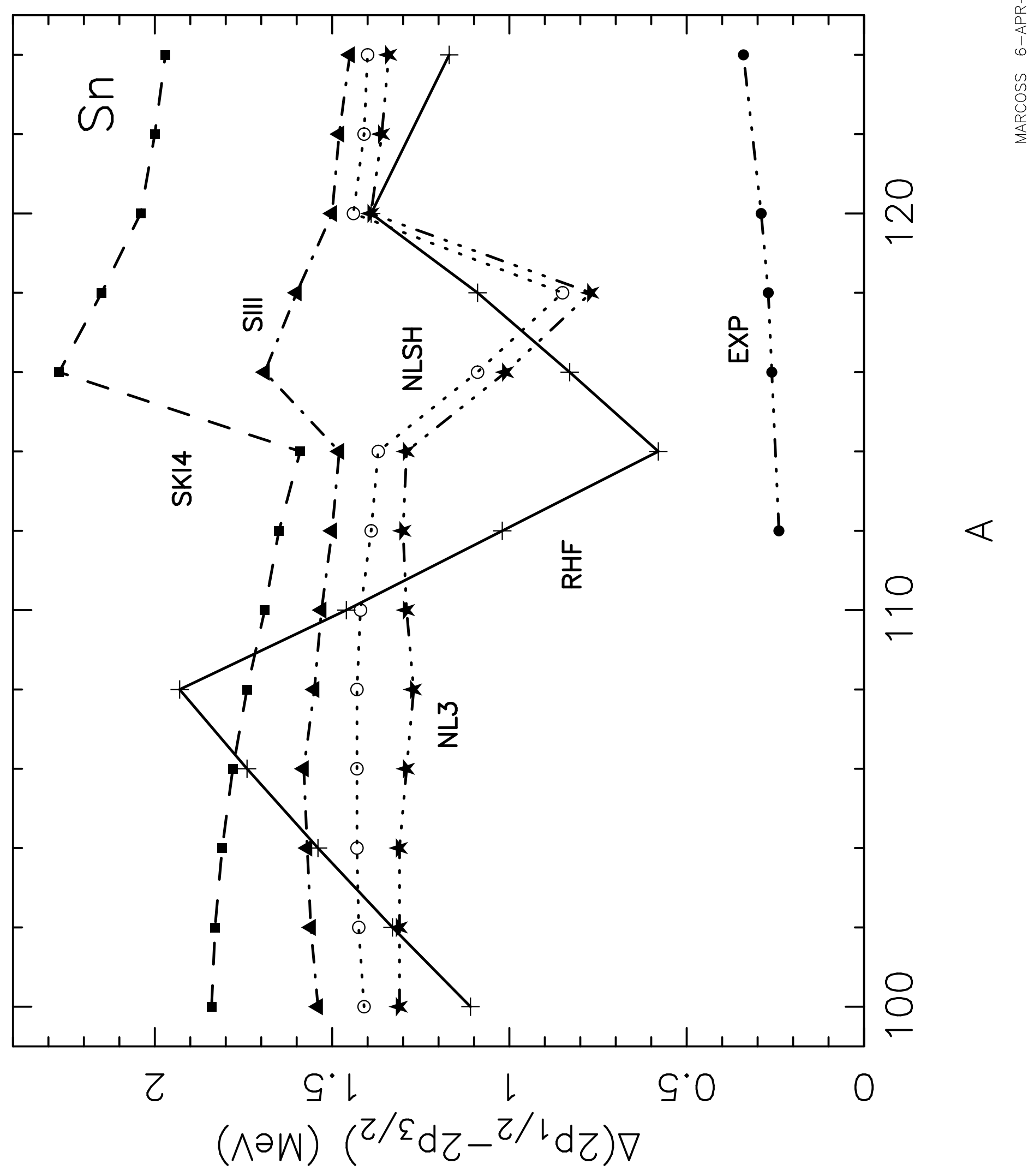




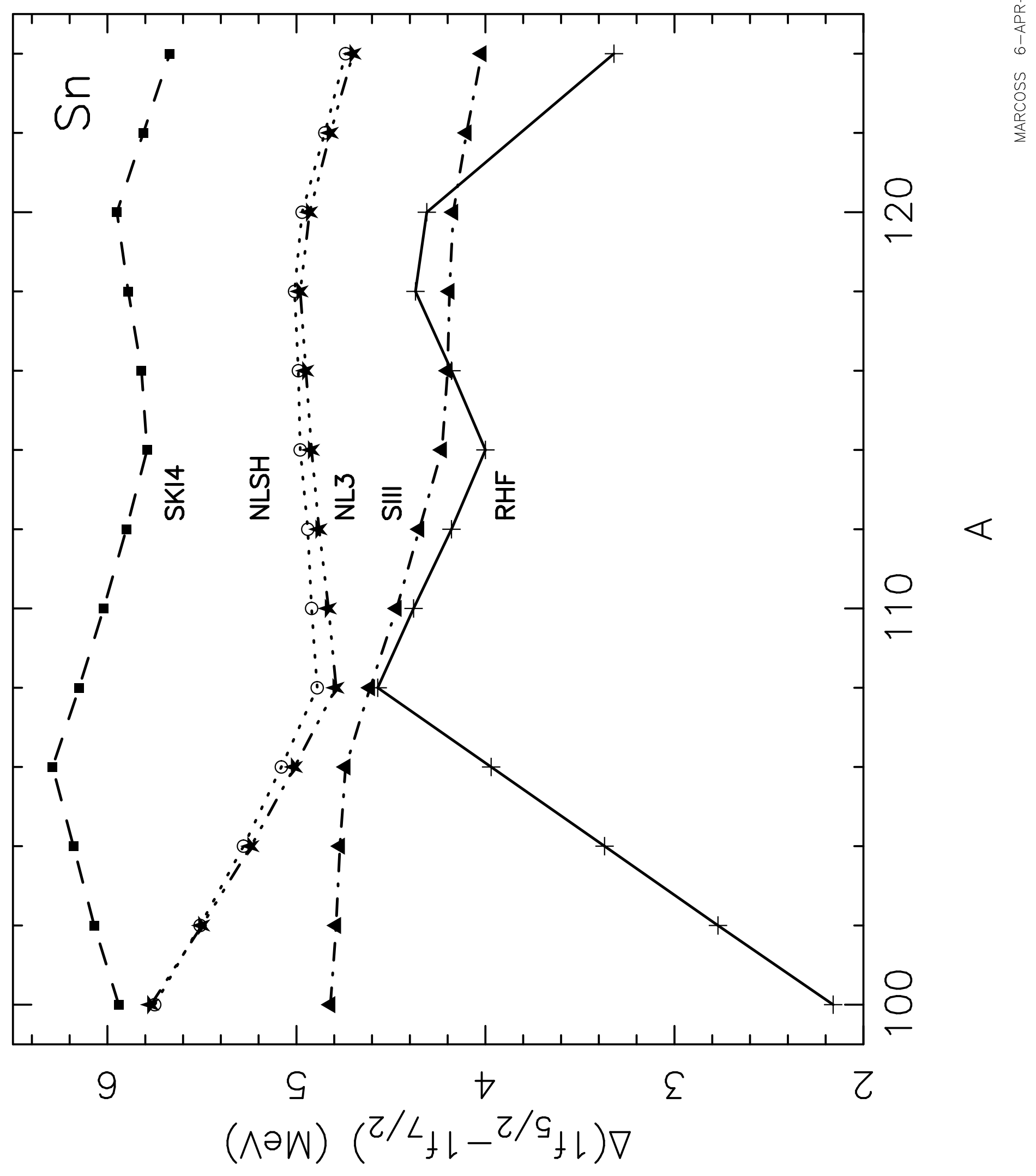

